\title{
Left Ventricular Dysfunction and Plasmatic NT-proBNP Are Associated with Adverse Evolution in Respiratory Syncytial Virus Bronchiolitis
}

\author{
Moises Rodriguez-Gonzalez $1,2, * \mathbb{1}$, Alvaro Antonio Perez-Reviriego ${ }^{1,2}$, \\ Ana Castellano-Martinez ${ }^{2,3} \mathbb{D}$, Simon Lubian-Lopez ${ }^{2,4}$ and Isabel Benavente-Fernandez ${ }^{2,4}$ (D) \\ 1 Paediatric Cardiology Division, Puerta del Mar University Hospital, 11009 Cadiz, Spain \\ 2 Biomedical Research and Innovation Institute of Cadiz (INiBICA), Research Unit, Puerta del Mar University \\ Hospital, University of Cadiz, 11009 Cadiz, Spain \\ 3 Paediatric Nephrology Division, Puerta del Mar University Hospital, 11009 Cadiz, Spain \\ 4 Neonatology Division, Puerta del Mar University Hospital, 11009 Cadiz, Spain \\ * Correspondence: doctromoisesrodriguez@gmail.com
}

Received: 27 June 2019; Accepted: 26 July 2019; Published: 27 July 2019

\begin{abstract}
Aim: To investigate whether the presence of left ventricular myocardial dysfunction (LVMD) assessed by Tei index (LVTX) impacts the outcomes of healthy infants with Respiratory Syncytial Virus Bronchiolitis (RSVB). To explore whether N-terminal pro-B-type natriuretic peptide (NT-proBNP) increases the accuracy of traditional clinical markers in predicting the outcomes. Methods: A single-centre, prospective, cohort study including healthy infants aged 1-12 months old admitted for RSVB between 1 October 2016 and 1 April 2017. All patients underwent clinical, laboratory and echocardiographic evaluation within $24 \mathrm{~h}$ of admission. Paediatric intensive care unit (PICU) admission was defined as severe disease. Results: We enrolled 50 cases of RSVB (median age of 2 (1-6.5) months; $40 \%$ female) and 50 age-matched controls. We observed higher values of LVTX in infants with RSVB than in controls $(0.42$ vs. $0.36 ; p=0.008)$. Up to nine $(18 \%)$ children presented with LVMD (LVTX > 0.5), with a higher incidence of PICU admission (89\% vs. 5\%; $p<0.001)$. The diagnostic performance of NT-proBNP in predicting LVMD was high (area under the receiver operator characteristic curve (AUC) 0.95, CI 95\% 0.90-1). The diagnostic yield of the predictive model for PICU admission that included NT-proBNP was excellent (AUC 0.945, CI 95\% 0.880-1), and significantly higher than the model without NT-proBNP $(p=0.026)$. Conclusions: LVMD could be present in healthy infants with RSVB who develop severe disease. NT-proBNP seems to improve traditional clinical markers for outcomes.
\end{abstract}

Keywords: respiratory syncytial virus; NT-proBNP; echocardiography; pulmonary hypertension; myocardial dysfunction; tissue doppler imaging; Tei index; biomarkers; infants

\section{Introduction}

Respiratory Syncytial Virus Bronchiolitis (RSVB) is the leading cause of lower respiratory infection and hospital admission among children up to 2 years of age worldwide [1]. Approximately 2-6\% cases of RSVB will develop a severe form of the disease, requiring admission to the paediatric intensive care unit (PICU) and mechanical ventilation (MV) [1,2]. RSVB constitutes approximately 13\% of all PICU admissions [2]. Current guidelines recognize the identification of specific risk factors (congenital heart disease (CHD), chronic lung disease (CLD), prematurity, etc.) and clinical evaluation as the best tools to assess severity, predict evolution and tailor management [3].

Cardiovascular involvement seems to be a relevant prognostic factor in RSVB. Cardiovascular complications are present in up to $9 \%$ of cases of RSVB and constitute the second most common 
extrapulmonary manifestation after infectious complications [4]. These events usually present in an abrupt and unexpected manner in children with severe RSVB, and infants with CHD are particularly susceptible to these complications and adverse outcomes [5]. Interestingly, nearly half of the children admitted to PICU with RSVB are healthy prior to the clinical event [2]. In these patients, the presence of acute lung injury secondary to RSVB can also lead to important cardiovascular effects, especially elevated pulmonary vascular resistance and overload on the right ventricle (RV) [6-8]. Moreover, previous studies assessing the plasma levels of cardiac troponin in RSVB suggest an underrecognized yet clinically significant incidence of myocardial damage in this population [9-11]. Furthermore, RV global dysfunction in ventilated healthy infants has been reported [12]. Recently, we found that mild to moderate forms of pulmonary hypertension $(\mathrm{PH})$ could impact the outcome of healthy infants with RSVB [13].

Adverse RV-LV interactions and left ventricle (LV) myocardial dysfunction (LVMD) are emerging as important determinants of $\mathrm{PH}$ outcomes. $\mathrm{PH}$ can induce complex changes in LV geometry and causes an abnormal relaxation and non-uniform contraction pattern in the LV wall, leading to LVMD [14-16]. However, most studies in healthy infants with RSVB found no abnormalities when assessing LVMD through conventional echocardiographic parameters [12,13,17-19]. Remarkably, there are no studies to date assessing LVMD in RSVB by more sensitive methods such as tissue Doppler imaging (TDI) echocardiography.

$\mathrm{N}$-terminal pro-B-type natriuretic peptide (NT-proBNP) is a hormone synthesized and released into the circulation by ventricular myocytes in response to pressure/volume overload and an increase in myocardial wall stress [20]. Elevated serum NT-proBNP levels have been defined as a powerful biomarker in the diagnosis of PH, and both LVMD and RV myocardial dysfunction (RVMD) secondary to pulmonary diseases [21-25]. Of note, we recently showed how NT-proBNP could be considered an adequate biomarker for $\mathrm{PH}$ in previously healthy infants with RSVB [13].

In this study, we aimed to investigate the presence of adverse RV-LV interactions and LVMD (assessed by TDI-echocardiography) in previously healthy infants with RSVB. We hypothesized that acute $\mathrm{PH}$ with RV pressure overload may indeed have a direct impact on LV performance. We also hypothesized that those infants with LVMD are prone to developing a more severe form of disease. Finally, we sought to test NT-proBNP as a biomarker for LVMD and explore whether NT-proBNP increases the accuracy of traditional clinical markers in predicting the severity of the disease.

\section{Materials and Methods}

\subsection{Design, Settings and Study Population}

This was a single-centre, prospective, cohort study including infants aged 1-12 months old admitted to the paediatric department in our institution (a tertiary university-affiliated hospital in Spain) due to RSVB (determined by a confirmed Respiratory Syncytial Virus (RSV) antigen test) between 1 October 2016 and 1 April 2017. All patients underwent clinical, laboratory and echocardiographic evaluation within $24 \mathrm{~h}$ of admission. We excluded patients with co-existing CHD or CLD, prematurity, those that received MV or intravenous fluid before assessment, and those with poor-quality echocardiographic images or incomplete medical records. Severe cases were screened for coinfection and, if present, were also excluded. The control group consisted of age-matched healthy infants who underwent evaluation for heart murmur at our paediatric cardiology outpatient clinic during the study period. These controls followed the same echocardiographic protocol as study patients. Our Institutional Review Board approved the study. Informed consent was obtained for all patients.

\subsection{Clinical and Laboratory Assessment and Outcomes}

The bronchiolitis score of Sant Joan de Déu (BROSJOD) [26] was used to assess severity at admission clinically. A BROSJOD score greater than 10 points is indicative of a severe clinical state. Venous $\mathrm{pH}$ and $\mathrm{pCO} 2$ were determined and respiratory acidosis (RA) was diagnosed when $\mathrm{pH}$ 
$<7.35$ and pCO2 > $45 \mathrm{mmHg}$. Plasma NT-proBNP levels at admission were determined using a commercially available electrochemiluminescent immunoassay kit (ElecSys 2010, Roche Diagnostics). The primary outcome was PICU admission during hospitalization. PICU admission criteria for RSVB at our institution include the presence of: apnea, extreme bradycardia, the need for respiratory support greater than high-flow nasal cannula oxygen therapy or inotropic support.

\subsection{Echocardiographic Assessment}

Standard techniques to obtain M-mode, two-dimensional and Doppler (color, pulsed, continuous and TDI) echocardiograms were performed by the same experienced paediatric cardiologist (RGM) as recommended in the guidelines for the paediatric echocardiogram [27]. Images were obtained using a Phillips IE33 ultrasound scanner with an 8 or 12-MHz sectorial transducer. Each examination was recorded, and all the studies were reviewed off-line by 2 observers (RGM and PRA), who were blinded to the patient's clinical profile. All echocardiographic measurements represent the average of 3 beats. Control and case echocardiographic data were deidentified before data analysis. Figures 1 and 2 show the main echocardiographic measures used in this study.
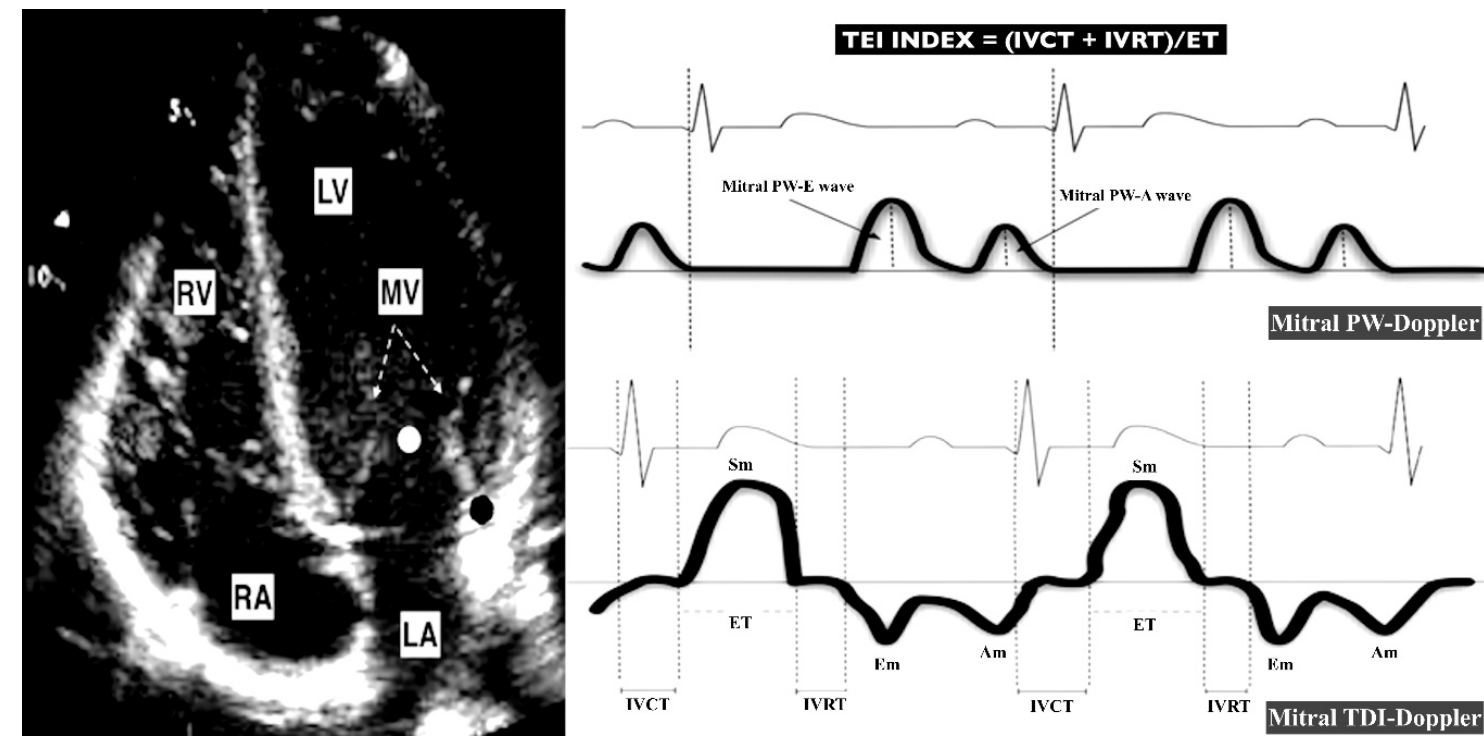

IVCT

IVRT

IVCT

IVRT Mitral TDI-Doppler

Figure 1. Echocardiographic parameters used for the estimation of LV performance. The left panel shows a four-chamber apical view. The right panel shows the waves used to assess LV performance. The Mitral PW-Doppler wave is obtained at the level of the LV inflow (white point). The Mitral TDI-Doppler wave is obtained at the level of the lateral mitral annulus (black point). MV (mitral valve; dotted arrow). RV (right ventricle). LV (left ventricle). RA (right atria). LA (left atria). PW (pulsed wave). DTI (Doppler tissue imaging). Sm (Doppler imaging (TDI)-derived peak systolic annular velocity). Em (TDI-derived early diastolic mitral annulus velocity). Am (TDI-derived late diastolic mitral annulus velocity). PW-E (PW-derived mitral peak early diastolic velocity). PW-A (PW-derived mitral peak late diastolic velocity). IVCT (TDI-derived isovolumic contraction time). IVRT (TDI-derived isovolumic relaxation time). ET (TDI-derived ejection time). 

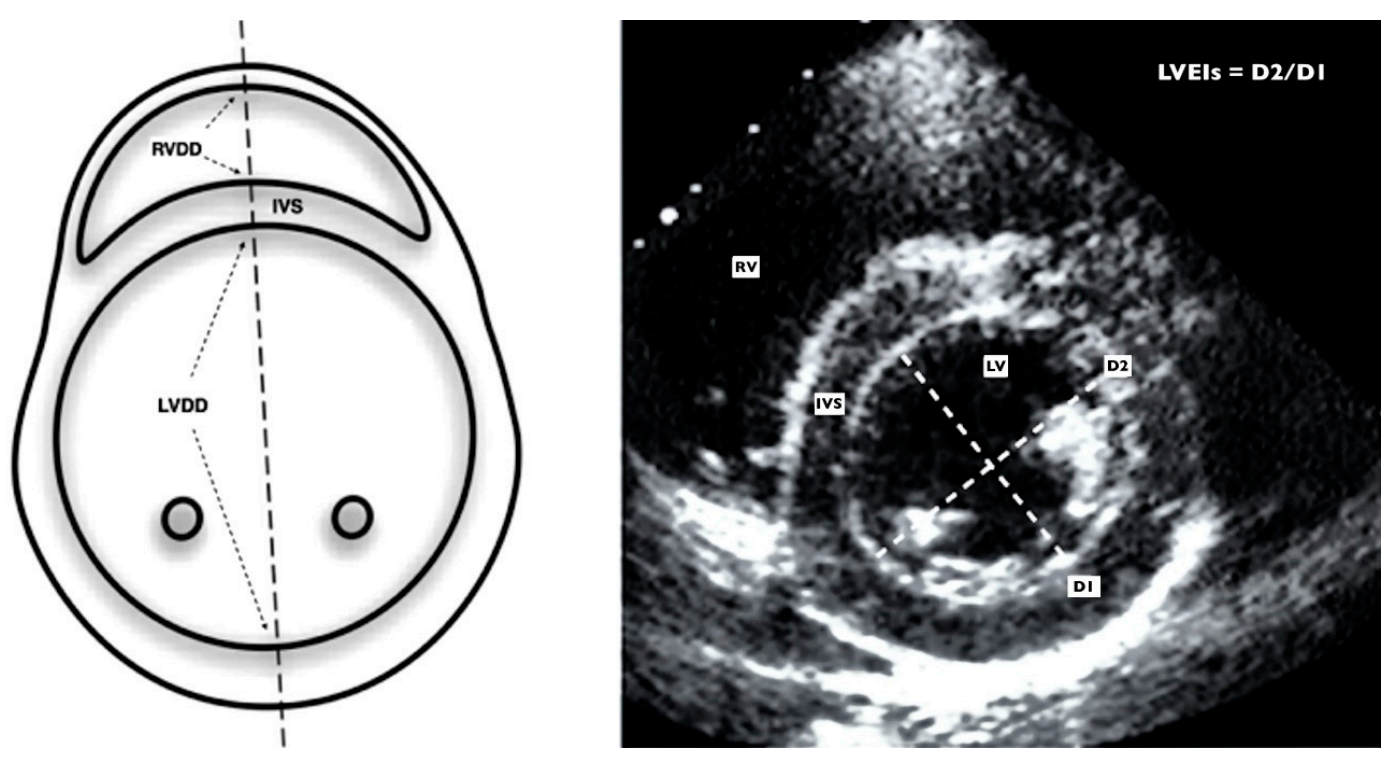

Figure 2. Echocardiographic parameters used for the estimation of RV and pulmonary hemodynamics. The figure shows a parasternal short axis view at the level of the papillary muscles. The left panel shows a schematic representation and the right panel shows real echocardiographic imaging. The LV systolic eccentricity index (LVEI) is obtained with de D2/D1 (dotted lines) ratio measured in systole as showed in the illustration. A LVEI $>1.2$ is suggestive of raised RV pressures or pulmonary hypertension in infants $^{32}$. RVDD (right ventricular diastolic diameter; dotted arrows). LVDD (left ventricular diastolic diameter; dotted arrows). IVS (Interventricular septum). RV (right ventricle). LV (left ventricle). D2 (LV systolic anteroposterior diameter). D1 (LV systolic laterolateral diameter).

\subsubsection{Right-Sided Echocardiographic Assessment}

The RV end-diastolic diameter (RVEDD) and the RV/LV ratio (RVLVr) were used as indicators of RV dilation. RV systolic function was assessed by the tricuspid annular plane systolic excursion (TAPSE) [28], and by the tissue Doppler imaging (TDI) derived peak systolic annular velocity at the tricuspid level of the RV free wall (St). RV Tei index (RVTX) was used as a measurement of global (systolic and diastolic) RV function. It was calculated using the TDI-derived isovolumic contraction (IVC), isovolumic relaxation (IVR) and ejection time (ET) intervals (measured at the lateral part of the tricuspid annulus) as previously described [29]. RV systolic pressure was calculated by the systolic gradient of the tricuspid regurgitation jet (TRJG) and using the simplified Bernoulli equation [30]. Due to the absence of an adequate tricuspid regurgitation jet in most patients, the RV outflow tract acceleration time/ejection time ratio (ATET) [31], the LV systolic eccentricity index (LVEI) [32], and the presence of septal flattening at the end of systole on the qualitative assessment were also used as indicators of RV systolic pressures [30]. The LVEI was also used as a measurement of leftward displacement of the interventricular septum (IVS).

\subsubsection{Left-Sided Echocardiographic Assessment}

The LV end-diastolic diameter (LVEDD) was used as an indicator of LV dilation. The LV shortening fraction (LVSF) and the tissue Doppler imaging (TDI)-derived peak systolic annular velocity at the mitral level of the LV free wall (Sm) were used to assess LV systolic function. To assess the diastolic LV function, we obtained the mitral peak early (E) and late (A) diastolic velocity using the pulsed wave Doppler of the mitral valve inflow. Also, the TDI-derived early diastolic mitral annulus velocity (Em) was measured at the lateral part of the mitral annulus. The E/Em ratio and E/A ratio were calculated as indicators of LV filling pressures. LV Tei index (LVTX) was used as a measurement of global (systolic and diastolic) LV function. It was calculated using the TDI-derived isovolumic contraction (IVC), isovolumic relaxation (IVR) and ejection time (ET) intervals (measured at the lateral part of the mitral 
annulus) as previously described [29]. Cui and Robertson reported in 2006 that the mean normal value of the LVTX for infants aged 1-12 months is 0.35 (0.09), and that a LVTX less than 0.5 is the upper limit of normal (2Zscore) [33]. Therefore, a LVTX > 0.5 was defined as LVMD in this study.

\subsection{Reproductibility}

To explore intra-observer and inter-observer agreement, 30 echocardiographic studies were randomly selected and analysed offline. To estimate the intra-observer agreement, the first observer (RGM) remeasured the LVTX, RVTX and LVEI with a 30-day interval blinded to previous measurements and patient information. To assess the inter-observer agreement, the means of both observers for each measurement were compared.

\subsection{Statistical Analysis}

Continuous data are presented as the median (range) or mean (standard deviation) after testing for normality with the Shapiro-Wilk test; categorical data are presented as frequencies and percentage. Mean comparison was performed using the Student's $t$ test or Wilcoxon Mann-Whitney test as appropriate. Proportions were compared using the Chi-square test or exact methods as necessary. Pearson and Spearman coefficients were used to assess correlations between continuous data. A receiver operator curve (ROC) analysis was used to determine the diagnostic accuracy of NT-proBNP for LVMD. The best cut-off value of NT-proBNP to detect LVMD was empirically estimated based on the Liu method, and values of sensitivity (Se), specificity (Sp), negative predictive value (NPV) and positive predictive value (PPV) were calculated for the obtained cut point. We selected 2 predefined predictive models for PICU admission. The selection of the included variables was based on the theoretical background and the exploratory analysis. Model 1 (proposed model) included age, BROSJOD score and NT-proBNP levels and model 2 (traditional model) included age and BROSJOD score. Prediction models were evaluated through multivariate logistic regression analysis. The discriminating ability of each model was assessed by the area under the receiver operator characteristic (AUC) curve. The AUCs from the obtained models were then compared by using the DeLong method [34] to determine whether any model resulted in increased predictive accuracy. The reliability of echocardiographic measurements was evaluated with the intra-class correlation (ICC) coefficients and Bland-Altman (BA) analysis [35]. Based on the 95\% confident interval of the ICC coefficients, values less than 0.5, between 0.5 and 0.75 , between 0.75 and 0.9 , and greater than 0.90 were considered indicative of poor, moderate, good, and excellent reliability, respectively. All the statistical analyses were performed using the Stata 13.0. (StataCorp. 2013. Stata Statistical Software: Release 13. College Station, TX: StataCorp LP.). A $p$ value $<0.05$ was considered statistically significant.

\section{Results}

\subsection{Baseline Characteristics and Outcomes of Patients with RSVB}

We enrolled a total of 50 cases of RSVB with a median age of 2 (1-6.5) months ( $40 \%$ female). The control group consisted of 50 healthy infants with no differences regarding age, sex or body surface area (BSA) distribution (Table 1). RSVB patients were admitted 2.76 (1.23) days after the initial symptoms, with a median BROSJOD score of 6 (1-14), a median SpO2 of 93\% (87-98\%), and a median heart rate of $118 \mathrm{bpm}(89-179 \mathrm{bpm})$. Up to nine (18\%) cases presented RA and a BROSJOD score $>10$. A total of $10(20 \%)$ cases needed PICU admission within 1.20 (0.38) days from hospitalization (length of PICU stay 5 (2-9) days) and were classified as having severe RSVB. A total of 3 of these 10 cases required MV and seven of them required continuous positive airway pressure (CPAP). No cases of arrhythmia different from sinus tachycardia were observed. No patient required inotropic support and none of the included patients died. 
Table 1. Baseline clinical and laboratory characteristics of the Respiratory Syncytial Virus Bronchiolitis (RSVB) population and comparison with controls.

\begin{tabular}{cccc}
\hline Variable & RSVB Group $(\boldsymbol{n}=\mathbf{5 0 )}$ & Control Group $(\boldsymbol{n}=\mathbf{5 0 )}$ & $p$ Value \\
\hline Age (months) & $2(1-6.5)$ & $2(1-9)$ & 0.591 \\
Female sex & $20(40)$ & $23(46)$ & 0.545 \\
BSA (m2) & $0.28(0.18-0.43)$ & $0.27(0.18-0.42)$ & 0.617 \\
Time of symptoms (days) & $2.76(1.23)$ & - & - \\
BROSJOD score & $6(1-14)$ & - & - \\
SpO2 (\%) & $93(87-98)$ & $99(95-100)$ & $<0.001$ \\
Heart rate (bpm) & $118(89-179)$ & - & -002 \\
pH & $7.36(7.22-7.45)$ & - & - \\
pCO2 & $42(31-71)$ & - & - \\
Nt-proBNP (pg/mL) & $511(62-5532)$ & - & - \\
Respiratory Acidosis & $9(18)$ & - & - \\
BROSJOD $>10$ & $9(18)$ & - & - \\
PICU admission & $10(20)$ & $-133)$ & - \\
\hline
\end{tabular}

BSA (body surface area); PICU (paediatric intensive care unit); BROSJOD score (bronchiolitis score of Sant Joan de Déu); Nt-proBNP (N-terminal pro-B-type natriuretic peptide); PICU (Paediatric intensive care unit).

\subsection{Echocardiographic Alterations in Patients with RSVB}

The RSVB group had a higher proportion of pericardial effusion ( $34 \%$ vs. $6 \% ; p<0.001)$. All cases of pericardial effusion were mild and did not require any treatment. We observed higher values of LVTX ( 0.42 vs. $0.36 ; p=0.008)$ in infants with RSVB than in controls (Figure 3). The RSVB group also presented more cases of septal flattening ( $28 \%$ vs. $6 \% ; p=0.003)$, and higher TRJG ( $27 \mathrm{vs.} 22 \mathrm{mmHg}$; $p=0.013)$, RVTX ( 0.39 vs. $0.36 ; p=0.005)$ and LVEI (1.08 vs. $1 ; p<0.001)$ than the control group. There were no differences between RSVB and control groups regarding echocardiographic parameters of ventricular dimensions, systolic or diastolic function (Table 2).

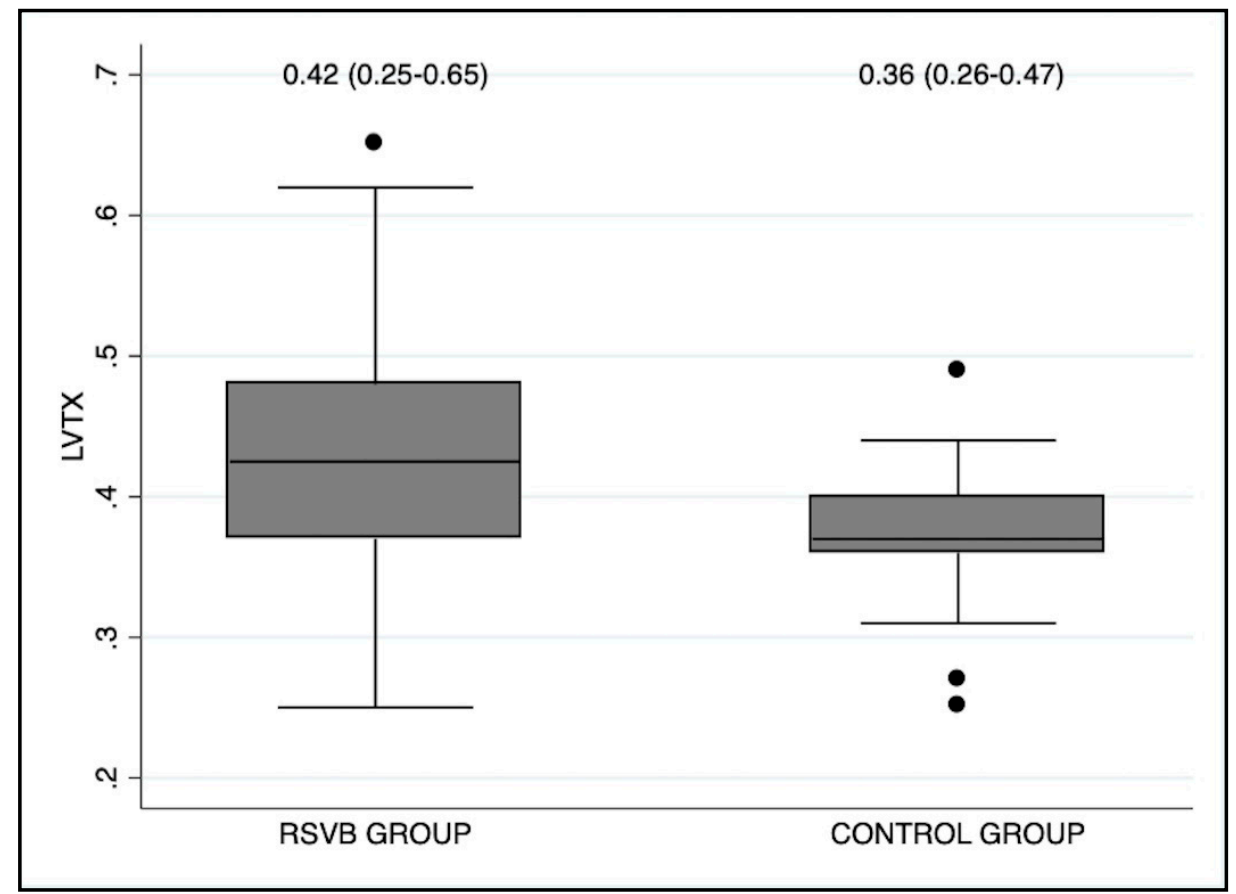

Figure 3. Box-plot diagram showing that the cases of RSVB presented higher values of LVTX at admission than healthy controls $(p=0.008)$. LVTX (Left ventricular Tei index). 
Table 2. Baseline echocardiographic characteristics of the RSVB population and comparison with controls.

\begin{tabular}{cccc}
\hline Variable & RSVB Group $(n=50)$ & Control Group $(n=50)$ & $p$ Value \\
\hline Pericardial effusion & $17(34)$ & $3(6)$ & $<0.001$ \\
Left ventricle & & & \\
LVDD (mm) & $21(17-29)$ & $21(14-28)$ & 0.470 \\
LVSF (\%) & $38.5(5.6)$ & $39(5)$ & 0.851 \\
Sm (cm/s) & $8.7(1.6)$ & $9(1.2)$ & 0.573 \\
Mitral E (cm/s) & $98(79-125)$ & $96(76-123)$ & 0.798 \\
Mitral A (cm/s) & $75(49-97)$ & $71(51-93)$ & 0.259 \\
Mitral E/A & $1.25(0.93-1.98)$ & $1.43(1-2)$ & 0.200 \\
Mitral Em (cm/s) & $9.4(6-5-14)$ & $10(6.6-15)$ & 0.110 \\
Mitral Am (cm/s) & $9(5-15)$ & $9(6-13)$ & 0.182 \\
Mitral E/Em (cm/s) & $10.2(5.8-20)$ & $9.8(5.2-17)$ & 0.283 \\
LVTX & $0.42(0.25-0.65)$ & & $0.36(0.26-0.47)$ \\
Right ventricle & & $10(7-14)$ & \\
RVDD (mm) & $10(7-19)$ & $0.46(0.28-0.76)$ & 0.264 \\
RV/LV ratio & $0.49(0.28-0.80)$ & $12(1.8)$ & 0.473 \\
TAPSE (mm) & $12.3(1.6)$ & $9.1(1.7)$ & 0.901 \\
St (cm/s) & $8.5(2)$ & $0.37(0.26-0.48)$ & 0.005 \\
RVTX & $0.39(0.25-0.65)$ & $30(60)$ & 0.633 \\
Adequate TRJ & $25(50)$ & $22(16-34)$ & 0.013 \\
TRJG (mmHg) & $27(18-47)$ & $0.39(0.03)$ & 0.288 \\
ATET & $0.38(0.06)$ & $1(0.95-1.12)$ & $<0.001$ \\
LVEI & $1.08(0.98-1.45)$ & $3(6)$ & 0.003 \\
Septal Flattening & $14(28)$ & &
\end{tabular}

LVDD (left ventricle diastolic diameter); LVSF (left ventricular shortening fraction); LVTX (left ventricular Tei index); RVDD (right ventricular diastolic diameter); RV (right ventricle); LV (Left ventricle); TAPSE (tricuspid annular plane systolic excursion); RVTX (Right ventricular Tei index), TRJ (tricuspid regurgitation jet); TRJG (tricuspid regurgitation jet gradient); ATET (right ventricular acceleration time/right ventricular ejection time ratio); LVEI (systolic left ventricular eccentricity index).

\subsection{Adverse LV-RV Interactions in Patients with RSVB}

In the RSVB group, increased LVTX was related to echocardiographic parameters indicating higher RV dimensions (Rho RVDD = 0.56, Rho RVLVr $=0.60)$, higher RV pressures (Rho TRJG $=0.54$, Rho ATET $=-0.50$, Rho LVEI $=0.77)$, and decreased RV global function (Rho RVTX=0.74). We did not find associations of echocardiographic measurements of RV systolic function (TAPSE and St) with LVTX (Table 3 and Figure 4).

Table 3. Correlation coefficients between LVTX and RV echocardiographic parameters and plasmatic NT-proBNP levels in RSVB cases.

\begin{tabular}{ccc}
\hline Variable & Rho & $p$ Value \\
\hline RVDD & 0.56 & 0.003 \\
RV/LV ratio & 0.60 & 0.001 \\
TAPSE & -0.19 & 0.159 \\
St & -0.12 & 0.217 \\
RVTX & 0.738 & $<0.001$ \\
TRJG & 0.54 & 0.004 \\
ATET & -0.50 & 0.009 \\
LVEI & 0.77 & $<0.001$ \\
NT-proBNP & 0.73 & $<0.001$
\end{tabular}

RVDD (right ventricular diastolic diameter); RV (right ventricle); LV (Left ventricle); TAPSE (tricuspid annular plane systolic excursion); RVTX (Right ventricular Tei index); TRJ (tricuspid regurgitation jet); TRJG (tricuspid regurgitation jet gradient); ATET (right ventricular acceleration time/right ventricular ejection time ratio); LVEI (systolic left ventricular eccentricity index). 

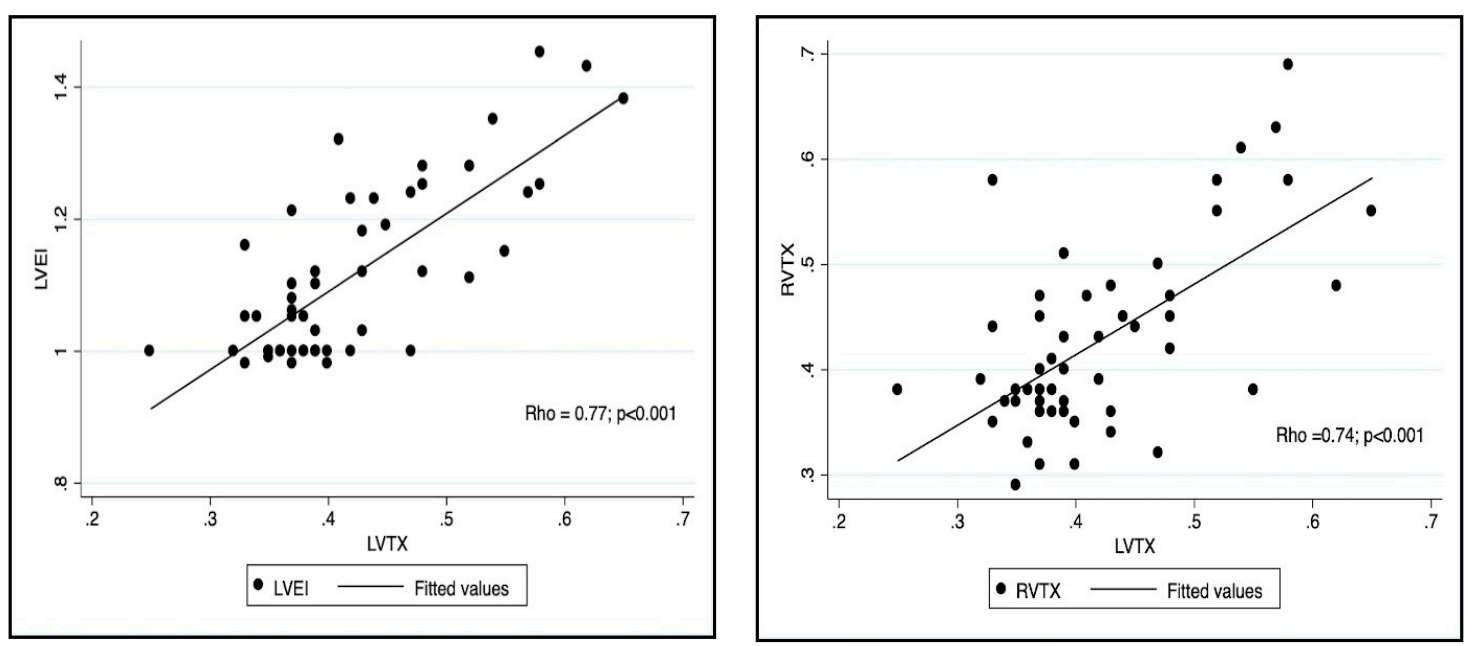

Figure 4. A representation of the correlation found between LVTX and parameters of RV function (RVTX, right panel) and RV pressures (LVEI, left panel). LVTX (Left ventricular Tei index). RVTX (right ventricular Tei index). LVEI (systolic left ventricular eccentricity index).

\subsection{LVMD in Patients with RSVB}

We found LVMD, defined by a LVTX > 0.5, in nine (18\%) patients with RSVB (Table 4). LVMD was associated with PICU admission ( $89 \%$ vs. $5 \% ; p<0.001)$. These patients presented with a higher BROSJOD score (11 vs. $6 ; p<0.001)$, lower SpO2 (90\% vs. $94 \% ; p<0.001)$, more cases of RA ( $55 \%$ vs. $9 \% ; p=0.001)$, and higher NT-proBNP levels $(2221 \mathrm{pg} / \mathrm{mL}$ vs. $377 \mathrm{pg} \mathrm{ml} ; p<0.001)$ than those with a normal LV myocardial function. The LVTX was also strongly correlated with NT-proBNP levels $($ Rho $=0.73)$ (Figure 5).

Table 4. Clinical and laboratory characteristics in patients with RSVB and LVMD, and comparison with those patients with normal LV function.

\begin{tabular}{cccc}
\hline Variable & LVMD $(\boldsymbol{n}=\mathbf{9} ; \mathbf{1 8} \%)$ & Normal LV Function $(\boldsymbol{n}=\mathbf{4 1 ;} \mathbf{8 2} \mathbf{\%})$ & $\boldsymbol{p}$ Value \\
\hline Age (months) & $2(1-4.5)$ & $2(1-6.5)$ & 0.538 \\
Female sex & $2(22)$ & $18(44)$ & 0.230 \\
BSA $\left(\mathrm{m}^{2}\right)$ & $0.29(0.18-0.33)$ & $0.28(0.18-0.43)$ & 0.742 \\
Time of symptoms (days) & $2(1.5)$ & $3(1-7)$ & 0.488 \\
BROSJOD score & $11(9-14)$ & $6(1-13)$ & $<0.001$ \\
SpO2 (\%) & $90(87-93)$ & $94(88-98)$ & $<0.001$ \\
Heart rate (bpm) & $135(100-164)$ & $116(89-179)$ & 0.141 \\
pH & $7.3(7.25-7.42)$ & $7.36(7.22-7.45)$ & 0.115 \\
pCO2 (mmHg) & $54(31-61)$ & $41(31-71)$ & 0.009 \\
Nt-proBNP (pg/mL) & $2221(891-5532)$ & $377(62-1779)$ & $<0.001$ \\
PICU admission & $8(89)$ & $2(5)$ & $<0.001$ \\
\hline
\end{tabular}

LVMD (left ventricular myocardial dysfunction); BSA (Body surface area); PICU (paediatric intensive care unit). 

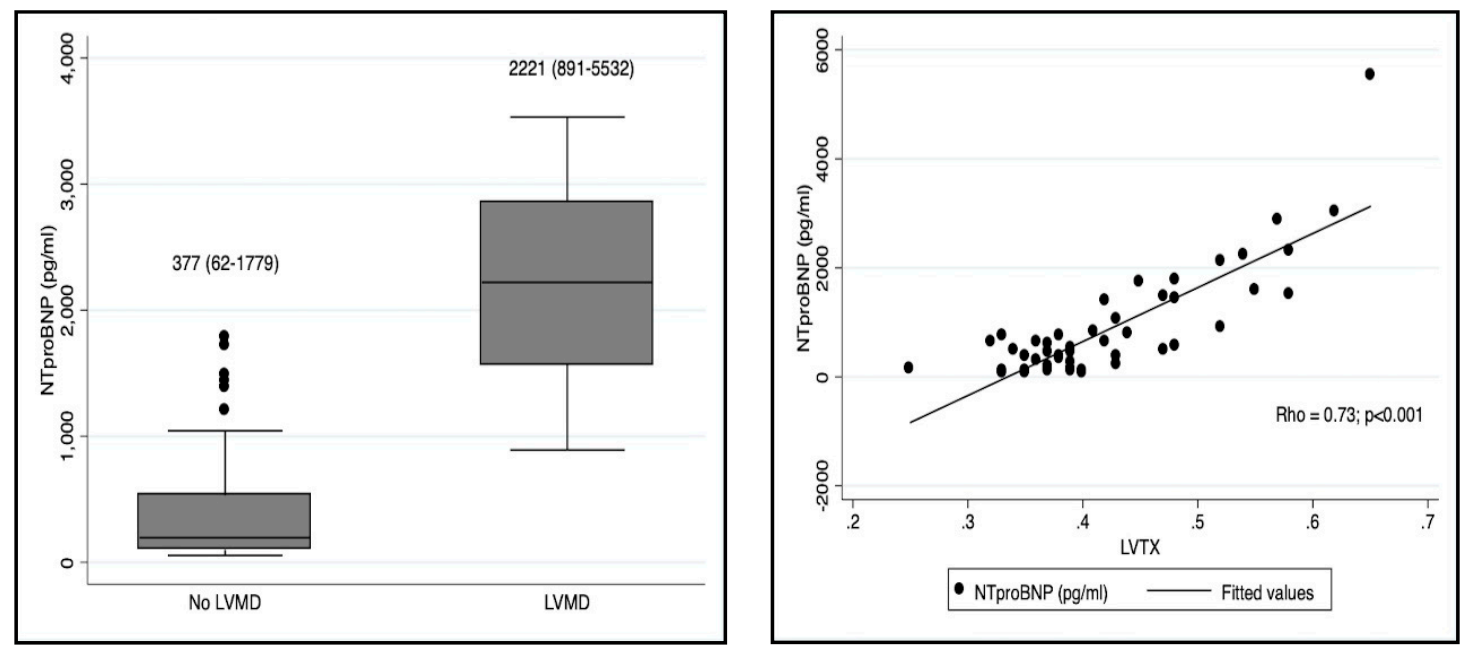

Figure 5. The left panel is a box-plot diagram representing the comparison of NT-proBNP levels between patients with and without LVMD. The right panel represents the correlation found between LVTX and NT-proBNP levels. LVTX (Left ventricular Tei index); LVMD (Left ventricular myocardial dysfunction).

\subsection{NT-ProBNP as Biomarker for LVMD}

The diagnostic performance of NT-proBNP to predict LVMD in infants with RSVB resulted in an area under the ROC curve of 0.91 (CI95\% 0.79-0.98) (Figure 6). The best estimated cut-off value to predict LVMD on echocardiography was $1500 \mathrm{pg} / \mathrm{mL}$, correctly classifying 92\% of cases, with a Se of 0.80 (CI95\% 0.49-0.94), Sp of 0.95 (CI95\% 0.83-0.98), a PPV of 0.80 (CI95\% 0.49-0.94), and an NPV of 0.95 (CI95\% 0.87-0.99) (Youden index 0.75).

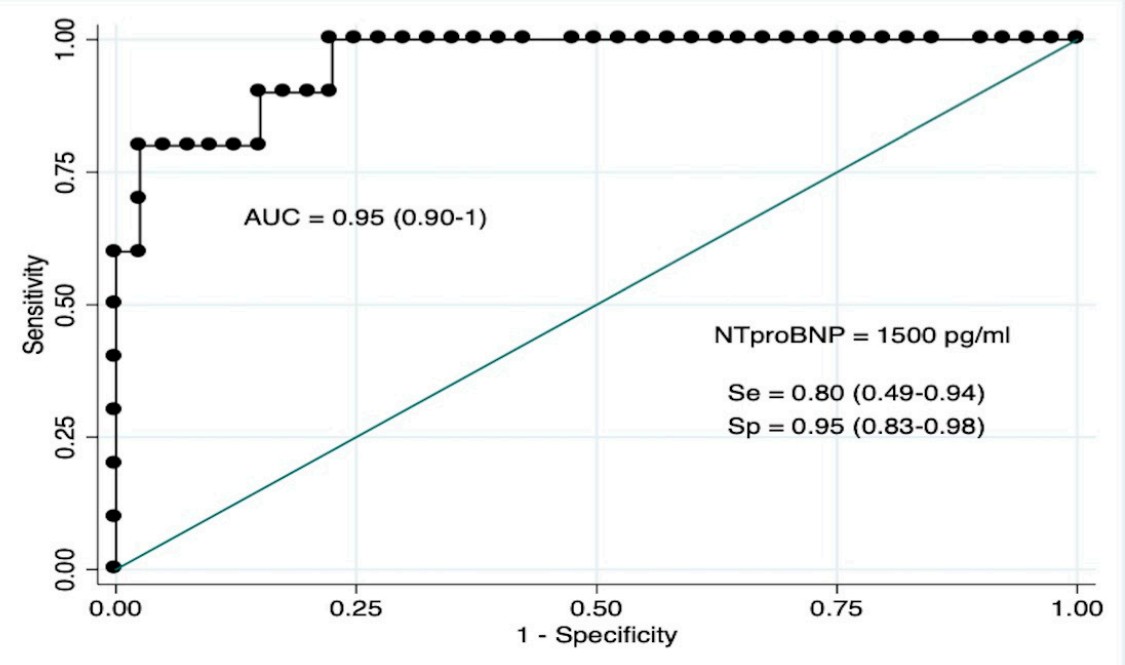

Figure 6. Representation of the receiver operating characteristic curve of NT-proBNP to detect LVMD in infants with RSVB. LVMD (Left ventricular myocardial dysfunction).

\subsection{Clinical and Laboratory Predictors of PICU Admission in RSVB}

We developed two different and predefined prediction models for PICU admission in RSVB. The variables used were clinical parameters that are traditionally used to assess severity in RSVB (age and clinical score), and NT-proBNP as biomarker for LVMD. Model 1 (the proposed model) included age $<3$ months, BROSJOD score $>10$, and NT-proBNP $>1500 \mathrm{pg} / \mathrm{mL}$. Model 2 (the traditional model) included age $<3$ months and BROSJOD score $>10$. The diagnostic yield of model 1 for PICU admission was excellent (AUC 0.945, CI95\% 0.880-1), and significantly higher than the yield for model $2(p=0.026)$. In 
model 1, the presence of NT-proBNP levels $>1500 \mathrm{pg} / \mathrm{mL}$ was the only independent predictive factor for PICU admission in RSVB, with an OR 27.03 (CI95\% (1.50-487), $p=0.025)$ (Table 5 and Figure 7).

Table 5. Univariate and Multivariate logistic regression analysis performed to find a predictive model for PICU admission during hospitalization in our RSVB cohort.

\begin{tabular}{cccccc}
\hline Variable & $\begin{array}{c}\text { Univariate Analysis } \\
\text { OR (CI 95\%) }\end{array}$ & $\boldsymbol{p}$ Value & $\begin{array}{c}\text { Multivariate Analysis } \\
\text { OR (CI 95\%) }\end{array}$ & $p$ Value & pseudoR2 \\
\hline Model 1 & & & & $<0.001$ & 0.51 \\
Age $<3$ months & $0.88(0.19-4.04)$ & 0.875 & $0.71(0.66-7.58)$ & 0.777 & \\
BROSJOD score $>10$ & $44.33(6.22-315.50)$ & $<0.001$ & $3.06(0.12-76.44)$ & 0.494 & \\
NT-proBNP $>1500$ pg/mL & $76(9.27-622)$ & $<0.001$ & $27.03(1.50-487)$ & 0.025 & 0.38 \\
Model 2 & & & & $<0.001$ & \\
Age $<3$ months & $0.88(0.19-4.04)$ & 0.875 & $0.52(0.06-4.21)$ & 0.541 & \\
BROSJOD score $>10$ & $44.33(6.22-315.50)$ & $<0.001$ & $49.27(6.38-380)$ & $<0.001$ & \\
\hline
\end{tabular}

OR (Odds ratio); CI (Confidence interval).

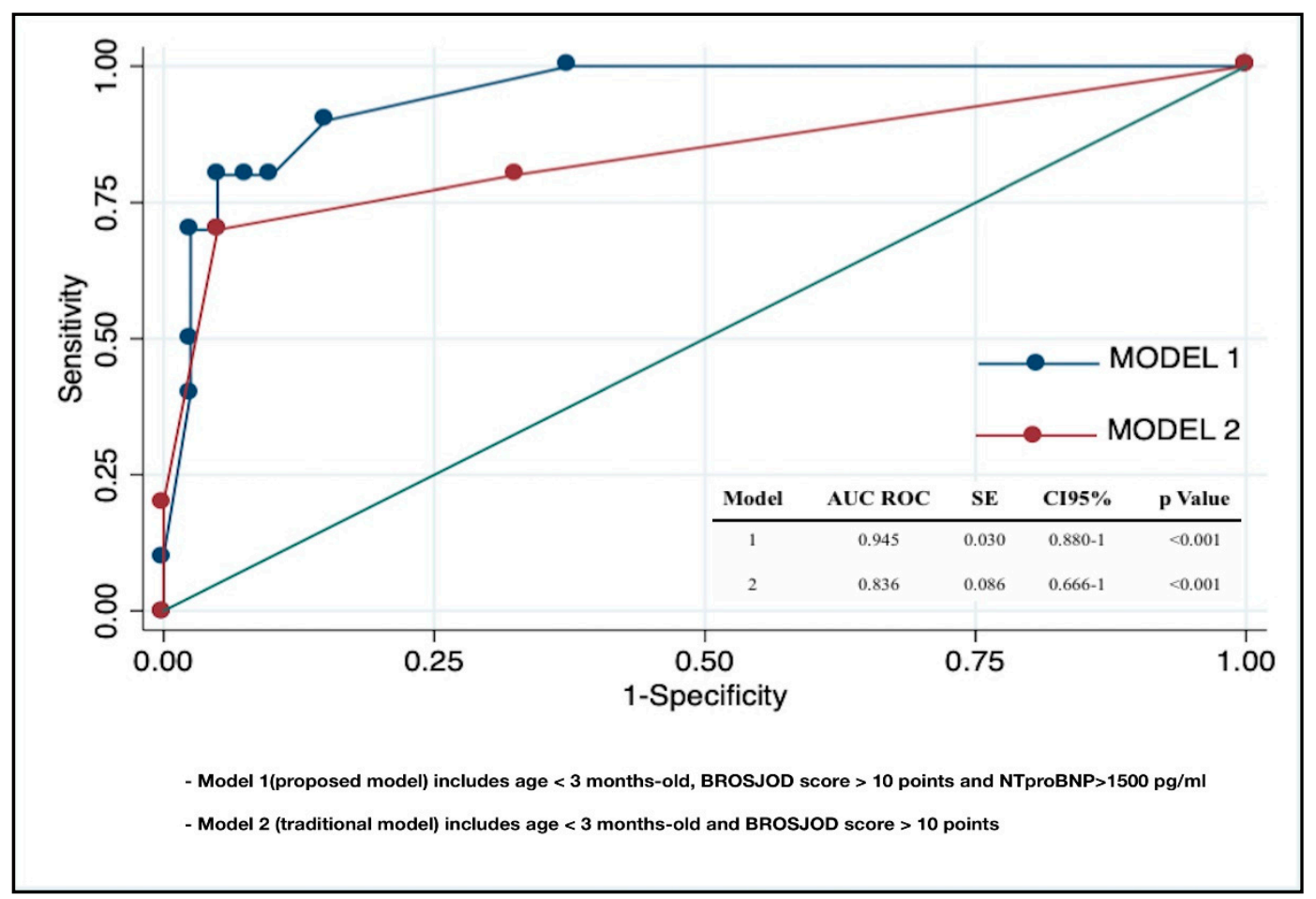

Figure 7. Graphical representation of the comparison between the areas under the receiver operator characteristic curves of the predictive models selected.

\subsection{Reproductibility}

The intra-observer and inter-observer agreement for LVTX, RVTX and LVEI were good or excellent, with all ICC coefficients > 0.75 (Table 6). 
Table 6. Intra-observer and inter-observer agreement scores for the main echocardiographic measurements performed in this study, the left ventricular and right ventricular Tei indexes (LVTX and RVTX), and the systolic left ventricular eccentricity index (LVEI).

\begin{tabular}{ccccc}
\hline \multirow{2}{*}{ Variable } & \multicolumn{2}{c}{ Intra-observer (RG.M) } & \multicolumn{2}{c}{ Inter-observer (RG.M and PR.A) } \\
\cline { 2 - 5 } & ICC (95\% CI) & BA (LOA) & ICC (95\% CI) & BA (LOA) \\
\hline LVTX & $0.95(0.93-0.99)$ & $0.00(-0.03-0.03)$ & $0.84(0.74-0.93)$ & $-0.01(-0.07-0.05)$ \\
RVTX & $0.97(0.95-0.99)$ & $-0.002(-0.02-0.02)$ & $0.86(0.77-0.95)$ & $-0.015(-0.07-0.04)$ \\
LVEI & $0.89(0.82-0.96)$ & $-0.007(-0.04-0.03)$ & $0.84(0.73-0.94)$ & $-0.01(-0.05-0.03)$ \\
\hline
\end{tabular}

ICC (interclass coefficient); CI (confidence interval); BA (Bland-Altman average); LOA (limits of agreement).

\section{Discussion}

The main finding of our study is that LVMD was observed at the early stages of the disease in up to $18 \%$ of previously healthy infants with RSVB when assessed by DTI-derived LVTX. The LVMD was associated with a more severe respiratory state, PICU admission, and echocardiographic signs of RV pressure overload and RVMD, indicating the presence of adverse RV-LV interactions in cases of severe RSVB. Also, we observed that NT-proBNP accurately identified LVMD. Moreover, we found an added benefit to the addition of NT-proBNP to the clinical evaluation in predicting the development of severe disease in this population.

CHD is an important cause of morbidity and mortality in RSVB [5]. This may be related to multiple physiological factors including baseline compromised cardiorespiratory function and the potential development of PH. However, little is known about LVMD and its association with RV function, pulmonary hemodynamics, and outcomes in previously healthy infants with RSVB. In accordance with the literature, the present study reveals that conventional parameters of myocardial function are not altered in RSVB [17-19]. Only one previous study has assessed myocardial performance using the TEI index in RSVB [12]. Our results are consistent with those reported by Thorburn et al. who found RVMD in ventilated patients with severe RSVB. However, they did not demonstrate any association between PH and RVMD. This may be due to the use of TRJG alone as an echocardiographic marker of $\mathrm{RV}$ pressure, and the presence of PH may had been underestimated. In a recent work from our group, we used a combination of different echocardiographic parameters to assess RV pressures and $\mathrm{PH}$ was reported in up to $22 \%$ of RSVB cases at early stages of the disease. In agreement with Thorburn et al., we did not find an association between PH and RV or LVMD [13]. We assessed ventricular function only by conventional parameters (TAPSE and LVSF).

To the best of our knowledge, this is the first study to evaluate LVMD using TDI-derived LVTX, and to assess RV-LV interactions in infants with RSVB. LVTX, which includes both systolic and diastolic time intervals to assess the global cardiac dysfunction, is an easily performable, recordable and reproducible parameter with normal reference values that can be applied to the entire spectrum of the paediatric population, regardless of age, heart rate, and BSA [36]. Using LVTX, we found LVMD in nearly $20 \%$ of cases. The RV shares muscle fibres, the inter-ventricular septum (IVS), and the pericardial sac with the LV. Consequently, changes in RV affect the LV, a concept termed ventricular interdependence $[16,37,38]$. In this study, we observed a moderate to strong correlation between LVTX and leftward displacement of the IVS (LVEI), raised RV pressures (TRGJ, ATET) and reduced RV global function (RVTX), confirming the presence of adverse RV-LV interactions in cases with severe RSVB. These results do not imply casualty, but in the absence of primary (myocarditis, cardiomyopathies, CHD) or secondary (sepsis, severe acidosis...) causes of LVMD, we suggest RV pressure overload and RV dysfunction due to pulmonary disease as the underlying condition for LVMD in our RSVB cohort. Recent paediatric studies have reported that $\mathrm{PH}$ can induce complex changes in LV geometry and causes an abnormal relaxation and non-uniform contraction pattern in the LV wall, leading to LVMD [14-16], supporting our hypothesis. These observations could add new insights into the pathophysiology of RSVB, highlighting a key role of the cardiovascular system, especially LV myocardial performance in 
this setting. Validating our findings in similar populations in different settings may also provide a basis to implement new therapeutic approaches for this disease, which currently has no effective treatment. Possible new therapeutic approaches may include the initiation of early respiratory support in cases with increased NT-proBNP, pulmonary vasodilators to reduce RV pressure overload and avoiding epinephrine in cases with LVMD.

In RSVB, the major goals are the prevention and early identification of infants at risk for severe disease in order to provide the best management options and decrease morbidity. Current guidelines recommend only clinical observation for this purpose in infants without known comorbidities [3]. However, most clinical scores for RSVB are not well validated and fail to predict outcomes [39,40]. Recently, the BROSJOD score, a validated clinical score for RSVB, has shown a strong capacity to predict the evolution in the course of RSVB, but it is not yet generalizable due to the single-centre character of the study [26]. In this context, the identification of novel biomarkers with adequate predictive value for disease severity in RSVB is an area of increasing research interest. Neutrophins, cytokines and leukotrienes are promising but not widely available for clinical practice [41]. Previous studies have also tested cardiac troponin as a prognostic marker with inconsistent results $[9,10,12,13]$. The LVMD found in our population was only identifiable by TDI-echocardiography, suggesting that it was mild in most of our patients. Nevertheless, this does not mean that LVMD is inconsequential in RSVB. Remarkably, most patients with LVMD presented with severe disease at admission and most of them required PICU admission. Of note, we included non-ventilated infants at early stages of the disease (mean time of 2.76 (1.23) days after the initial symptoms), when the patients had not yet been admitted to the PICU, increasing the prognostic value of our results. Therefore, assessing and understanding myocardial function in RSVB seems to be relevant.

Another interesting finding of our study was that NT-proBNP could be a useful biomarker for LVMD and subsequent outcomes in RSVB. Previous studies have also documented the correlation between LVTX and NT-proBNP plasma levels [42-44]. We explored the diagnostic accuracy of NT-proBNP to detect LVTX > 0.50, which was high (AUC 0.91), with an optimal cut-off value of 1500 pg/mL (Se 0.80, Sp 0.95, PPV 0.80, NPV 0.95). We also tested the benefit in adding NT-proBNP to the currently used clinical data to assess outcomes in RSVB. Although the predictive models including age and BROSJOD score presented a high predictive accuracy for PICU admission in our population, we observed that the addition of NT-proBNP to this model increased the predictive value significantly, and that NT-proBNP was the only independent factor within the analysed values that predicted a severe course of the disease. Most cases of RSVB are mild forms manageable on an outpatient basis without the need for laboratory exams. Nevertheless, many children sufficiently ill to require hospitalization will routinely have laboratory studies drawn. Adding NT-proBNP measurement to these studies could be useful in order to identify high-risk patients who benefit from echocardiographic screening to $\mathrm{PH}$, RVMD or LVMD. Based on our results, it might be reasonable to perform an echocardiogram in those patients with NT-proBNP levels $>1500 \mathrm{pg} / \mathrm{mL}$.

This work included some limitations. Our study was performed at a single centre and with a relatively small size. We excluded irritable or unstable patients, where the technical difficulties due to respiratory comorbidity and the patient's inability to tolerate the evaluation could impact the results. The PICU admission criteria and, subsequently, the definition of severe diseases in the study are based on the protocol of our hospital, which can vary between institutions. Therefore, a larger multicentre cohort study including irritable or unstable cases and with uniform PICU admission criteria may be needed for the verification and generalization of our results. Finally, the assessment of myocardial function and PH on echocardiography was not confirmed by an independent gold-standard method, such as cardiac magnetic resonance imaging or right heart catheterization, and therefore some patients could have been misclassified. 


\section{Conclusions}

Adverse RV-LV interactions and LVMD could be present in healthy infants with RSVB during the early stages of the disease, negatively impacting the outcome. NT-proBNP seems to be an adequate biomarker for LVMD. Adding NT-proBNP to traditional clinical markers to assess outcomes in RSVB could improve the early detection of those cases that will develop a severe illness. Future research is need in order to confirm these results and to design new therapeutic approaches based on them.

Author Contributions: Conceptualization, M.R.-G.; methodology, M.R.-G. and I.B.-F.; software, M.R.-G. and I.B.-F.; validation, M.R.-G., I.B.-F., A.A.P.-R., A.C.-M., S.L.-L.; formal analysis, M.R.-G. and I.B.-F.; investigation, M.R.-G., A.A.P.-R. and A.C.-M.; resources, M.R.-G. and S.L.-L.; data curation, M.R.-G., A.C.-M. and A.A.P.-R.; writing-original draft preparation, M.R.-G.; writing—review and editing, M.R.-G., I.B.-F., A.A.P.-R., A.C.-M., S.L.-L.; visualization, M.R.-G. and I.B.-F.; supervision, M.R.-G. and I.B.-F.; project administration, M.R.-G.

Funding: This research received no external funding.

Conflicts of Interest: The authors declare no conflict of interest.

\section{References}

1. Hasegawa, K.; Tsugawa, Y.; Brown, D.F.M.; Mansbach, J.M.; Camargo, C.A. Trends in bronchiolitis hospitalizations in the United States, 2000-2009. Pediatrics. Am. Acad. Pediatr. 2013, 132, 28-36.

2. Ghazaly, M.; Nadel, S. Characteristics of children admitted to intensive care with acute bronchiolitis. Eur. J. Pediatr. 2018, 177, 913-920. [CrossRef] [PubMed]

3. Ralston, S.L.; Lieberthal, A.S.; Meissner, H.C.; Alverson, B.K.; Baley, J.E.; Gadomski, A.M.; Johnson, D.W.; Light, M.J.; Maraga, N.F.; Mendonca, E.A.; et al. Clinical practice guideline: The diagnosis, management, and prevention of bronchiolitis. Am. Acad. Pediatr. 2014, 134, e1474-e1502. [CrossRef] [PubMed]

4. Willson, D.F.; Landrigan, C.P.; Horn, S.D.; Smout, R.J. Complications in infants hospitalized for bronchiolitis or respiratory syncytial virus pneumonia. J. Pediatr. 2003, 143, S142-S149. [CrossRef]

5. Tulloh, R.M.; Medrano-Lopez, C.; Checchia, P.A.; Stapper, C.; Sumitomo, N.; Gorenflo, M.; Bae, E.J.; Juanico, A.; Gil-Jaurena, J.M.; Wu, M.H.; et al. CHD and respiratory syncytial virus: Global expert exchange recommendations. Cardiol. Young 2017, 27, 1504-1521. [CrossRef] [PubMed]

6. Fitzgerald, D.; Davis, G.M.; Rohlicek, C.; Gottesman, R. Quantifying pulmonary hypertension in ventilated infants with bronchiolitis: A pilot study. J. Paediatr. Child Health 2001, 37, 64-66. [CrossRef] [PubMed]

7. Bardi-Peti, L.; Ciofu, E.P. Bronchiolitis and pulmonary hypertension. Pneumologia 2010, 59, 95-100. [PubMed]

8. Kimura, D.; McNamara, I.F.; Wang, J.; Fowke, J.H.; West, A.N.; Philip, R. Pulmonary hypertension during respiratory syncytial virus bronchiolitis: A risk factor for severity of illness. Cardiol. Young 2019, 20, 1-5. [CrossRef] [PubMed]

9. Clark, S.J.; Eisenhut, M.; Sidaras, D.; Hancock, S.W.; Newland, P.; Thorburn, K. Myocardial injury in infants ventilated on the paediatric intensive care unit: A case control study. Crit. Care 2006, 10, R128. [CrossRef]

10. Moynihan, J.A.; Brown, L.; Sehra, R.; Checchia, P.A. Cardiac troponin I as a predictor of respiratory failure in children hospitalized with respiratory syncytial virus (RSV) infections: A pilot study. Am. J. Emerg. Med. 2003, 21, 479-482. [CrossRef]

11. Thorburn, K. Cardiac Troponin T levels and myocardial involvement in children with severe respiratory syncytial virus lung disease. Acta Paediatr. 2004, 93, 887-890.

12. Thorburn, K.; Eisenhut, M.; Shauq, A.; Narayanswamy, S.; Burgess, M. Right ventricular function in children with severe respiratory syncytial virus (RSV) bronchiolitis. Minerva Anestesiol. 2011, 77, 46-53. [PubMed]

13. Rodriguez-Gonzalez, M.; Benavente Fernández, I.; Castellano-Martinez, A.; Lechuga-Sancho, A.M.; Lubián-López, S.P. NT-proBNP plasma levels as biomarkers for pulmonary hypertension in healthy infants with respiratory syncytial virus infection. Biomark. Med. 2019, 3. [CrossRef] [PubMed]

14. Driessen, M.M.P.; Meijboom, F.J. Adverse ventricular-ventricular interactions in right ventricular pressure load: Insights from pediatric pulmonary hypertension versus pulmonary stenosis. Physiol. Rep. 2016, 4, e12833. [CrossRef] [PubMed]

15. Burkett, D.A.; Slorach, C.; Patel, S.S.; Redington, A.N.; Ivy, D.D.; Mertens, L.; Younoszai, A.K.; Friendberg, M.K. Impact of Pulmonary Hemodynamics and Ventricular Interdependence on Left Ventricular Diastolic Function in Children with Pulmonary Hypertension. Circ. Cardiovasc. Imaging 2016, 9, e004612. [CrossRef] [PubMed] 
16. Motoji, Y.; Tanaka, H.; Fukuda, Y.; Sano, H.; Ryo, K.; Imanishi, J.; Miyoshi, T.; Sawa, T.; Mochizuki, Y.; Matsumoto, K.; et al. Interdependence of right ventricular systolic function and left ventricular filling and its association with outcome for patients with pulmonary hypertension. Int. J. Cardiovasc. Imaging 2015, 23, 691-698. [CrossRef]

17. Pahl, E.; Gidding, S.S. Echocardiographic assessment of cardiac function during respiratory syncytial virus infection. Pediatrics 1988, 81, 830-834. [PubMed]

18. Esposito, S.; Salice, P.; Bosis, S.; Ghiglia, S.; Tremolati, E.; Tagliabue, C.; Gualtieri, L.; Barbier, P.; Galeone, C.; Marchisio, P.; et al. Altered cardiac rhythm in infants with bronchiolitis and respiratory syncytial virus infection. BMC Infect Dis. 2010, 24, 305. [CrossRef]

19. Horter, T.; Nakstad, B.; Ashtari, O.; Solevåg, A.L. Right and left ventricular function in hospitalized children with respiratory syncytial virus infection. Infect. Drug Resist. 2017, 10, 419-424. [CrossRef]

20. Martinez-Rumayor, A.; Richards, A.M.; Burnett, J.C.; Januzzi, J.L., Jr. Biology of the Natriuretic Peptides. Am. J. Cardiol. 2008, 101, S3-S8. [CrossRef]

21. Kate ten, C.A.; Tibboel, D.; Kraemer, U.S. B-type natriuretic peptide as a parameter for pulmonary hypertension in children. A systematic review. Eur. J. Pediatr. 2015, 23, 1267-1275. [CrossRef] [PubMed]

22. Takatsuki, S.; Wagner, B.D.; Ivy, D.D. B-type natriuretic peptide and amino-terminal pro-B-type natriuretic peptide in pediatric patients with pulmonary arterial hypertension. Congenit Heart Dis. 2012, 7, 259-267. [CrossRef] [PubMed]

23. Hauser, J.A.; Demyanets, S.; Rusai, K.; Goritschan, C.; Weber, M.; Panesar, D.; Rindler, L.; Taylor, A.M.; Marculescus, R.; Burch, M.; et al. Diagnostic performance and reference values of novel biomarkers of paediatric heart failure. Heart 2016, 27, 1633-1639. [CrossRef] [PubMed]

24. Mladosievicova, B.; Urbanova, D.; Radvanska, E.; Slavkovsky, P.; Simkova, I. Role of NT-proBNP in detection of myocardial damage in childhood leukemia survivors treated with and without anthracyclines. J. Exp. Clin. Cancer Res. 2012, 11, 1. [CrossRef] [PubMed]

25. Li, Q.; Cui, C.-Y.; Zhang, C.; Guo, S.-Y.; Zhang, Q. On the changes of NT-proBNP level in children having undergone radical operation of tetralogy of Fallot and the clinical significance. Eur. Rev. Med. Pharmacol. Sci. 2015, 19, 3018-3022. [PubMed]

26. Balaguer, M.; Alejandre, C.; Vila, D.; Esteban, E.; Carrasco, J.L.; Cambra, F.J.; Jordan, I. Bronchiolitis Score of Sant Joan de Déu: BROSJOD Score, validation and usefulness. Pediatr. Pulmonol. 2017, 52, 533-539. [CrossRef] [PubMed]

27. Lai, W.W.; Geva, T.; Shirali, G.S.; Frommelt, P.C.; Humes, R.A.; Brook, M.M.; Pignatelli, R.H.; Rychik, J. Guidelines and Standards for Performance of a Pediatric Echocardiogram: A Report from the Task Force of the Pediatric Council of the American Society of Echocardiography. J. Am. Soc. Echocardiogr. 2006, 19, 1413-1430. [CrossRef] [PubMed]

28. Koestenberger, M.; Ravekes, W.; Everett, A.D.; Stueger, H.P.; Heinzl, B.; Gamillscheg, A.; Cvirn, G.; Boysen, A.; Fandl, A.; Nagel, B. Right ventricular function in infants, children and adolescents: Reference values of the tricuspid annular plane systolic excursion (TAPSE) in 640 healthy patients and calculation of $\mathrm{z}$ score values. J. Am. Soc. Echocardiogr. 2009, 22, 715-719. [CrossRef] [PubMed]

29. Tei, C.; Dujardin, K.S.; Hodge, D.O.; Bailey, K.R.; McGoon, M.D.; Tajik, A.J.; Seward, S.B. Doppler echocardiographic index for assessment of global right ventricular function. J. Am. Soc. Echocardiogr. 1996, 9, 838-847. [CrossRef]

30. Koestenberger, M.; Apitz, C.; Abdul-Khaliq, H.; Hansmann, G. Transthoracic echocardiography for the evaluation of children and adolescents with suspected or confirmed pulmonary hypertension. Expert consensus statement on the diagnosis and treatment of paediatric pulmonary hypertension. The European Paediatric Pulmonary Vascular Disease Network, endorsed by ISHLT and D6PK. Heart 2016, 102, ii14-ii22. [PubMed]

31. Habash, S.; Laser, K.T.; Moosmann, J.; Reif, R.; Adler, W.; Glöckler, M.; Kececioglu, D.; Dittrich, S. Normal values of the pulmonary artery acceleration time (PAAT) and the right ventricular ejection time (RVET) in children and adolescents and the impact of the PAAT/RVET-index in the assessment of pulmonary hypertension. Int. J. Cardiovasc. Imaging 2019, 35, 295-306. [CrossRef] [PubMed]

32. Abraham, S.; Weismann, C.G. Left Ventricular End-Systolic Eccentricity Index for Assessment of Pulmonary Hypertension in Infants. Echocardiography 2016, 16, 910-915. [CrossRef] [PubMed] 
33. Cui, W.; Roberson, D.A. Left ventricular Tei index in children: Comparison of tissue Doppler imaging, pulsed wave Doppler, and M-mode echocardiography normal values. J. Am. Soc. Echocardiogr. 2006, 19, 1438-1445. [CrossRef] [PubMed]

34. DeLong, E.R.; DeLong, D.M.; Clarke-Pearson, D.L. Comparing the areas under two or more correlated receiver operating characteristic curves: A nonparametric approach. Biometrics 1988, 44, 837-845. [CrossRef] [PubMed]

35. Koo, T.K.; Li, M.Y. A Guideline of Selecting and Reporting Intraclass Correlation Coefficients for Reliability. Res. J. Chiropr. Med. 2016, 15, 155-163. [CrossRef] [PubMed]

36. Cui, W.; Roberson, D.A.; Chen, Z.; Madronero, L.F.; Cuneo, B.F. Systolic and diastolic time intervals measured from Doppler tissue imaging: Normal values and Z-score tables, and effects of age, heart rate, and body surface area. J. Am. Soc. Echocardiogr. 2008, 21, 361-370. [CrossRef] [PubMed]

37. Burkett, D.A.; Slorach, C.; Patel, S.S.; Redington, A.N.; Ivy, D.D.; Mertens, L.; Younoszai, A.K.; Friendberg, M.K. Left Ventricular Myocardial Function in Children with Pulmonary Hypertension: Relation to Right Ventricular Performance and Hemodynamics. Circ. Cardiovasc. Imaging 2015, 8, e003260. [CrossRef]

38. Haeck, M.L.A.; Höke, U.; Marsan, N.A.; Holman, E.R.; Wolterbeek, R.; Bax, J.J.; Schalij, M.J.; Vliegen, H.W.; Delgado, V. Impact of right ventricular dyssynchrony on left ventricular performance in patients with pulmonary hypertension. Int. J. Cardiovasc. Imaging 2014, 30, 713-720. [CrossRef]

39. Bekhof, J.; Reimink, R.; Brand, P.L.P. Systematic review: Insufficient validation of clinical scores for the assessment of acute dyspnoea in wheezing children. Paediatric. Respiratory Rev. 2014, 15, 98-112. [CrossRef]

40. Destino, L.; Weisgerber, M.C.; Soung, P.; Bakalarski, D.; Yan, K.; Rehborg, R.; Wagner, D.R.; Gorelick, M.H.; Simpson, P. Validity of respiratory scores in bronchiolitis. Hosp. Pediatr. 2012, 2, 202-209. [CrossRef]

41. Brown, P.M.; Schneeberger, D.L.; Piedimonte, G. Biomarkers of respiratory syncytial virus (RSV) infection: Specific neutrophil and cytokine levels provide increased accuracy in predicting disease severity. Paediatric. Respiratory Rev. 2015, 16, 232-240. [CrossRef] [PubMed]

42. Takeuchi, D.; Saji, T.; Takatsuki, S.; Fujiwara, M. Abnormal tissue doppler images are associated with elevated plasma brain natriuretic peptide and increased oxidative stress in acute Kawasaki disease. Circ. J. 2007, 71, 357-362. [CrossRef] [PubMed]

43. Ozde, C.; Dogru, M.; Ozde, Ş.; Kayapinar, O.; Kaya, A.; Korkmaz, A. Subclinical right ventricular dysfunction in intermittent and persistent mildly asthmatic children on tissue Doppler echocardiography and serum NT-proBNP: Observational study. Pediatr. Int. 2018, 60, 1024-1032. [CrossRef] [PubMed]

44. Mikkelsen, K.V.; Møller, J.E.; Bie, P.; Ryde, H.; Videbaek, L.; Haghfelt, T. Tei index and neurohormonal activation in patients with incident heart failure: Serial changes and prognostic value. Eur. J. Heart Failure 2006, 8, 599-608. [CrossRef] [PubMed]

(C) 2019 by the authors. Licensee MDPI, Basel, Switzerland. This article is an open access article distributed under the terms and conditions of the Creative Commons Attribution (CC BY) license (http://creativecommons.org/licenses/by/4.0/). 